\title{
Application of Cas12j for Streptomyces editing and cluster activation
}

Lee Ling Tan a, Elena Heng a , Nadiah Zulkarnain ${ }^{a}$, Chung Yan Leong ${ }^{\text {, }}$, Veronica Ng ${ }^{\text {, }}$ Lay

Kien Yang ${ }^{b}$, Deborah Chwee San Seow ${ }^{b}$, Guangrong Peh ${ }^{c}$, Yee Hwee Lim ${ }^{c}$, Koduru

Lokanand $^{\mathrm{a}}$, Yoganathan Kanagasundaram ${ }^{\mathrm{b}}$, Siew Bee $\mathrm{Ng}^{\mathrm{b}}$ and Fong Tian Wong ${ }^{\mathrm{a}, \mathrm{b}}$

${ }^{\text {a }}$ Molecular Engineering Lab, Institute of Molecular and Cell Biology, A*STAR, Singapore

${ }^{\mathrm{b}}$ Singapore Institute of Food and Biotechnology Innovation, A*STAR, Singapore

${ }^{\mathrm{c}}$ Functional Molecules \& Polymers, Institute of Chemical and Engineering Sciences, A*STAR, Singapore

To whom correspondence may be addressed:

wongft@,imcb.a-star.edu.sg, 61 Biopolis Drive, Proteos, 138673, Singapore

\begin{abstract}
In recent years, CRISPR-Cas toolboxes for Streptomyces editing have rapidly accelerated natural product discovery and engineering. However, Cas efficiencies are also oftentimes strain dependent, subsequently a variety of Cas proteins would allow for flexibility and enable genetic manipulation within a wider range of Streptomyces strains. In this work, we have further expanded the Cas toolbox by presenting the first example of Cas $12 \mathrm{j}$ mediated editing in Streptomyces sp. A34053. In our study, we have also observed significantly improved editing efficiencies with Acidaminococcus sp. Cas12j compared to Cas12a, Francisella tularensis subsp. novicida U112's type V-A Cas (FnCpf1).
\end{abstract}

Keywords: CRISPR-Cas, Streptomyces, genome editing, Cas12 


\section{Introduction}

Actinomycetes are highly productive factories of bioactive natural products (NP) $[1,2]$. The activation and production of these pharmaceutically important, secondary metabolites are typically triggered by environmental signals [3]. Unfortunately, under laboratory conditions, it is estimated that $80 \%$ of this chemical diversity is silent [4-6]. To this end, various methods to activate these silent biosynthetic gene clusters were investigated and deployed [7]. In our laboratory and others, CRISPR-Cas mediated editing of actinomycetes, in particular Streptomyces, have accelerated NP discovery and engineering [8-10].

Previously, Streptococcus pyogenes Cas9 (SpCas9), Staphylococcus aureus Cas9 (SaCas9), Streptococcus thermophilus CRISPR 1 Cas9 (Sth1Cas9) and Francisella tularensis subsp. novicida U112's type V-A Cas (FnCpf1) have been used for genome editing in Streptomyces [8]. Cpf1, also known as Cas12a, has been shown to be highly efficient (75 - 95\%) for precise genome editing in the presence of a homology repair template in Streptomyces [11]. This is comparable to the more commonly used SpCas9. In 2020, Pausch et al. reported the discovery and usage of the hypercompact Cas12 sub family Acidaminococcus sp. Cas12j (AsCas12j) where the Cas protein has a single RuvC endonuclease domain for both processing of crRNA and cleavage [12]. Although it has similar functions and capabilities as Cas12a, Cas $12 \mathrm{j}$ is significantly smaller at 757 amino acid long, as compared to SpCas9 (1368 aa) and FnCpf1 (1300 aa). The AsCas12j system was demonstrated to be active in vitro and in human and plant cells.

Out of the three AsCas12j examined, AsCas12j-2 was reported to be self-processing and have high levels of editing efficiencies. Subsequently, we selected AsCas12j-2 for editing in Streptomyces 
sp. A34053 from the A*STAR Natural organism library (NOL) [13]. In this study, we demonstrated that AsCas12j-2 is functional in Streptomyces and in these examples, has even outperformed that of FnCpf1. This is the first reporting for the successful application of Cas $12 \mathrm{j}$ in genome manipulation of Streptomyces.

\section{Methods}

\section{Conjugation and screening of strains}

Spore preparations, conjugation protocol as well as the method for the screening of edits were adapted from Yeo et al, 2019 [8].

To obtain the spores of Streptomyces sp. A34053, the strain is first propagated in SV2 media (For $1 \mathrm{~L}$, add $15 \mathrm{~g}$ glucose, $15 \mathrm{~g}$ glycerol, $15 \mathrm{~g}$ soya peptone (Biobasic), $1 \mathrm{~g} \mathrm{CaCO}_{3}$ to deionized water, pH 7.0) and plated onto ISP Medium No. 4 (BD Biosciences). Spores were removed and resuspended in sterile TX buffer (50 mM Tris $\mathrm{pH} 7.4,0.001 \%(\mathrm{v} / \mathrm{v})$ Triton $\mathrm{X})$. Intergeneric conjugation between Streptomyces sp. A34053 and DNA methylase deficient WM3780 E. coli donor cells were performed by transforming promoter knock-in constructs into WM3780.

Apramycin-sensitive clones were picked for screening by colony PCR. Successful PCR amplicons were screened for the presence of the promoter or promoters via restriction enzyme digest. Positive samples were then validated with Sanger sequencing. 


\section{Fermentation and analysis}

Wild type Streptomyces sp. A34053 and edited mutants with promoter knock-in were cultured in 5 mL SV2 media for 3 - 5 days. Saturated seed cultures were diluted into fresh fermentation media: SV2, ISP2 (For $1 \mathrm{~L}$, add $10 \mathrm{~g}$ malt extract (Sigma-Aldrich), $4 \mathrm{~g}$ yeast extract (BD Biosciences), and 4 g glucose.), and CA08LB (For $1 \mathrm{~L}, 15 \mathrm{~g}$ glucose, $20 \mathrm{~g}$ cane molasses, $40 \mathrm{~g}$ soluble starch, 8 $\mathrm{g} \mathrm{CaCO}_{3}$, and $25 \mathrm{~g}$ cottonseed flour (Sigma-Alrich), $\mathrm{pH}$ adjusted to $\mathrm{pH}$ 7.2.) in a 1:20 volume ratio and fermented with $200 \mathrm{rpm}$ shaking at $30{ }^{\circ} \mathrm{C}$ in the dark. After 9 days, the cultures were pelleted then the separated biomass and supernatant were lyophilized. The dried samples were extracted by methanol then filter through filter paper (Whatman Grade 4) and filtrates were reconstituted for analysis.

The extracts were analysed on an Agilent 1290 Infinity LC System coupled to an Agilent 6540 accurate-mass quadrupole time-of-flight (QTOF) mass spectrometer. $5 \mu \mathrm{L}$ of extract was injected onto a Waters Acquity UPLC BEH C18 column, 2.1 x $50 \mathrm{~mm}, 1.7 \mu \mathrm{m}$. Mobile phases were water (A) and acetonitrile (B), both with $0.1 \%$ formic acid. The analysis was performed at flow rate of $0.5 \mathrm{~mL} / \mathrm{min}$, under gradient elution of $2 \% \mathrm{~B}$ to $100 \% \mathrm{~B}$ in $8 \mathrm{~min}$. Both MS and MS/MS data were acquired in positive electrospray ionization (ESI) mode. The typical QTOF operating parameters were as follows: sheath gas nitrogen, $12 \mathrm{~L} / \mathrm{min}$ at $325^{\circ} \mathrm{C}$; drying gas nitrogen flow, $12 \mathrm{~L} / \mathrm{min}$ at $350{ }^{\circ} \mathrm{C}$; nebulizer pressure, $50 \mathrm{psi}$; nozzle voltage, $1.5 \mathrm{kV}$; capillary voltage, $4 \mathrm{kV}$. Lock masses in positive ion mode: purine ion at $m / z 121.0509$ and HP-0921 ion at $m / z 922.0098$. 


\section{Results}

\section{Design of $12 \mathrm{j}$ vector}

To utilize AsCas12j-2, the backbone of an all-in-one pCRISPomyces-2 plasmid was used (Addgene \#61737 [14]), where the Cas protein was replaced with codon optimized AsCas12j-2 from Twist Biosciences (USA). The crRNA sequence [12], "5GTCGGAACGCTCAACGATTGCCCCTCACGAGGGGAC-3”, was inserted after the promoter transcribing the guide RNA. The general annotation of the elements in this plasmid is shown in Figure 1A.

\section{Functional Cas12j in comparison with Cas12a}

Since FnCpfl and AsCas12j-2 belong to the Type V CRISPR-Cas system and recognize similar PAM sites (TTN), and FnCpf1 was observed to be significantly successful in previous experiments [8], in this study, we will be comparing the genome editing capabilities of AsCas12j-2 with FnCpf1.

Three different clusters within Streptomyces sp. A34053 (Figure 1B) were targeted for promoter exchange to enable cluster activation [15]. Since FnCfp1 and AsCas12j-2 uses the same PAM site, the same protospacer ( $24 \mathrm{bps)}$ and homology flanks were used for both Cas proteins. In the editing of all three clusters, AsCas12j-2 was functional and was able to insert the new promoter (Table 1). The edited strains also demonstrated changes in chemical profiles with the promoter exchange (Figure 2). This would be the first example of Cas12j in Streptomyces. Interestingly, both AsCas12j-2 and FnCpf1 gave similar numbers of exconjugants but AsCas12j-2 consistently has a significantly high rate of edits whilst FnCpf1 yield no edited strains (Table 1). This suggests that 
although AsCas12j-2 and FnCpf1 have similar toxicity within Streptomyces sp. A34053, AsCas12j-2 outperforms FnCpf1 in genome editing, within the context of these clusters.
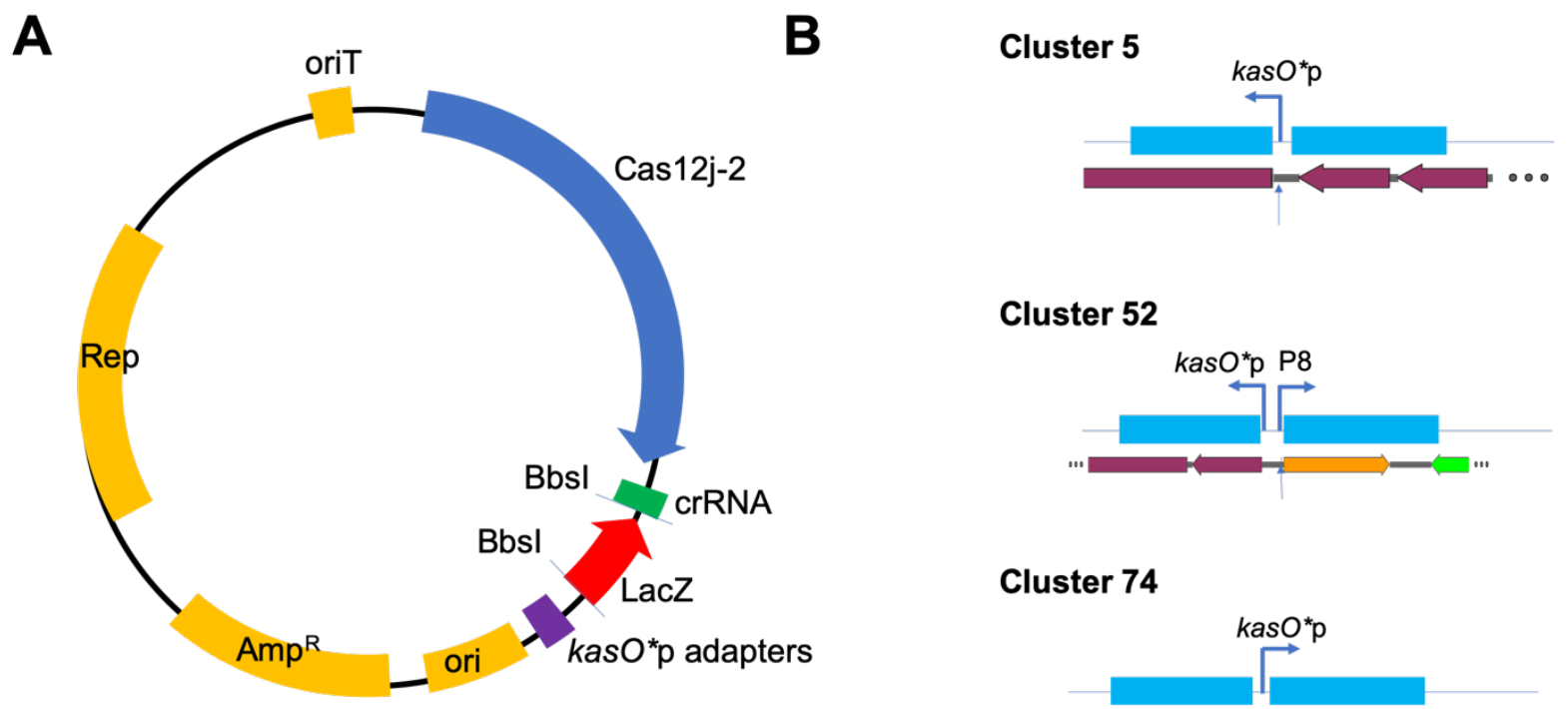

Cluster 52

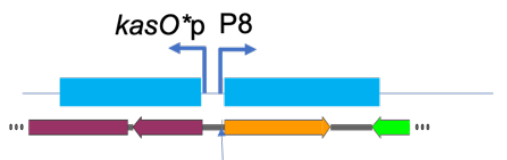

Cluster 74

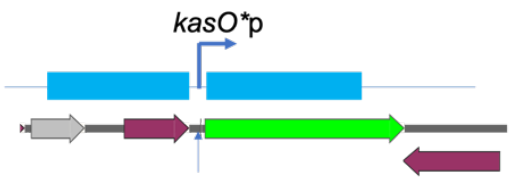

Figure 1. (A) General map of all-in-one editing CRISPR-Cas construct for one-step genome editing of Streptomyces using AsCas12j-2 along with (B) schematic homology flanks used for Streptomyces sp. A34053 editing.

(A) Rep: Replicon, AmpR: aparamycin resistance cassette, ori: origin of replication, oriT: origin of transfer, LacZ: LacZ operon for screening, kas $O^{*} \mathrm{p}$ adapters for addition of homology flanks, crRNA: CRISPR-RNA, BbsI sites for golden gate assembly of protospacer.

(B) Homology flanks (Blue) with insert of promoters are designed as the figure, where each flank is $\sim 2 \mathrm{~kb}$ in length and a $97 \mathrm{bps}\left(k a s O^{*} \mathrm{p}\right)$ or $778 \mathrm{bps}\left(k a s O^{*} \mathrm{p}\right.$ with $\mathrm{P} 8$ promoter) is inserted at the specified sites. The sites of the protospacer are also indicated on the genome with arrows. Legend 
of genes on the genome annotation; Grey: domain of unknown function, orange: transporter, green: regulator, red: biosynthetic enzymes.

Table 1. Editing efficiencies of AsCas12j-2 and FnCpf1

\begin{tabular}{|c|c|c|c|c|}
\hline Cluster & Cas protein & Insertion (kb) & Efficiency $^{\text {a }}$ & Exconjugants $^{\mathbf{b}}$ \\
\hline 5 & AsCas12j-2 & 0.1 & $50 \%(3 / 6)$ & $>50$ \\
& FnCpf1 & 0.1 & $0 \%(0 / 8)$ & $>50$ \\
\hline 74 & AsCas12j-2 & 0.1 & $75 \%(3 / 4)$ & 4 \\
\hline $52^{\mathrm{c}}$ & AsCas12j-2 & 1 & $0 \%(0 / 6)$ & 7 \\
\hline
\end{tabular}

a Editing efficiency given by the number of correct clones validated by sanger sequencing over the total number of clones picked (Supplementary Figure 1).

b Number of exconjugants observed per $100 \mu \mathrm{L}$ of spore preparation used in each conjugation. A typical spore prep contains $\sim 10^{6}-10^{7}$ spores $/ \mathrm{mL}$ as determined by serial dilution plating.

c Genome editing using FnCpf1 was not performed for this cluster. 


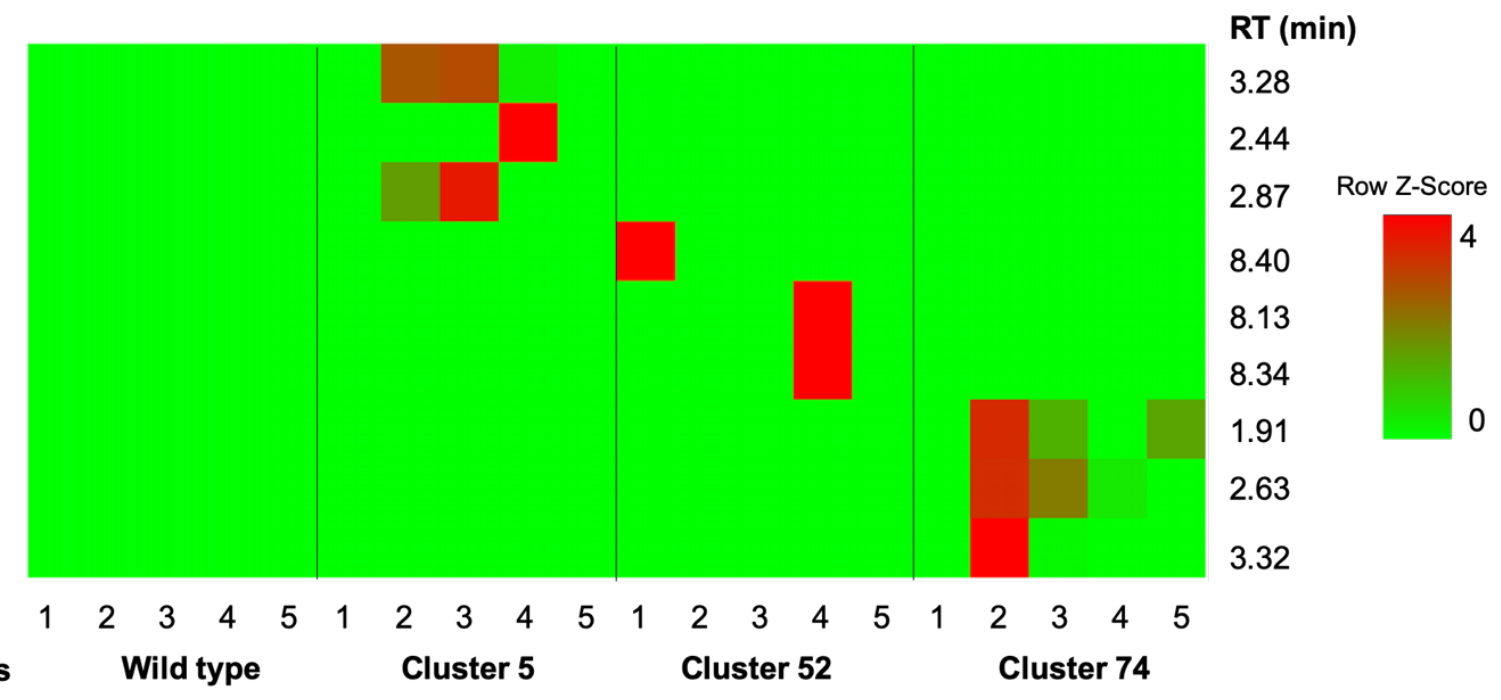

Figure 2. Heat map representation of secondary metabolites production between wild type and edited mutants for Cluster 5, 52 and 74 (Table 1). The comparison was done across 5 culture media (1: CA02LB, 2: CA07LB, 3: CA08LB, 4: CA09LB, 5: CA10LB). The heat map is generated by shinyheatmap interface [17] and scaled across rows.

\section{Discussion}

To harness the full biosynthetic potential of Streptomyces, much efforts have been placed in expanding the Cas toolbox for activation of silent biosynthetic gene clusters. The Cas protein, its associated levels of toxicity, its PAM recognition sites, as well as its efficiency of editing the genome are all important factors of consideration in its application in Streptomyces [16]. Here we report the successful application of Cas12j for Streptomyces usage. AsCas $12 \mathrm{j}-2$ is not only functional in Streptomyces sp. A34053, but it also has a higher editing efficiency compared to FnCpf1 in this study.

We believe that the hypercompact AsCas12j-2 will be a valuable tool in our strategy to generating genomic perturbations in Streptomyces. Previous studies have also shown successful applications 
of various CRISPR-Cas strategies from Streptomyces to the rare Actinomycetes without modifications or addition of helper proteins. In this same manner, we predict that the usage of Cas $12 \mathrm{j}-2$ could also be applied across the Actinomycetes family.

\section{Acknowledgement}

This work is supported by the National Research Foundation (NRF) $19^{\text {th }}$ Competitive Research

Program (CRP) grant - NRF-CRP19-2017-05. 


\section{References:}

[1] Barka, E.A., Vatsa, P., Sanchez, L., Gaveau-Vaillant, N., Jacquard, C., Klenk, H.P., Clément, C., Ouhdouch, Y., van Wezel, G.P., 2016. "Taxonomy, physiology, and natural products of actinobacteria." Microbiology and Molecular Biology Reviews, 80.1, 1-43.

[2] Genilloud, O., 2017. “Actinomycetes: Still a source of novel antibiotics.” Natural Product Reports, 34.10, 1203-32.

[3] Onaka, H., Mori, Y., Igarashi, Y., Furumai, T., 2011. "Mycolic acid-containing bacteria induce natural-product biosynthesis in Streptomyces species." Applied and Environmental Microbiology, $77.2,400-406$.

[4] Bentley, S.D., Chater, K.F., Cerdeño-Tárraga, A.M., Challis, G.L., Thomson, N.R., James, K.D., Harris, D.E., Quail, M.A., Kieser, H., Harper, D., Bateman, A., Brown, S., Chandra, G., Chen, C.W., Collins, M., Cronin, A., Fraser, A., Goble, A., Hidalgo, J., Hornsby, T., Howarth, S., Huang, C.H., Kieser, T., Larke, L., Murphy, L., Oliver, K., O’Neil, S., Rabbinowitsch, E., Rajandream, M.A., Rutherford, K., Rutter, S., Seeger, K., Saunders, D., Sharp, S., Squares, R., Squares, S., Taylor, K., Warren, T., Wietzorrek, A., Woodward, J., Barrell, B.G., Parkhill, J., Hopwood, D.A., 2002. “Complete genome sequence of the model actinomycete Streptomyces coelicolor A3(2).” Nature, 417.6885, 141-47.

[5] Challis, G.L., Ravel, J., 2000. "Coelichelin, a new peptide siderophore encoded by the Streptomyces coelicolor genome: Structure prediction from the sequence of its non-ribosomal peptide synthetase.” FEMS Microbiology Letters, 187.2, 111-14.

[6] Challis, G.L., 2008. "Genome mining for novel natural product discovery." Journal of Medicinal Chemistry, 51.9, 2618-28. 
[7] Scherlach, K., Hertweck, C., 2009. “ Triggering cryptic natural product biosynthesis in microorganisms." Organic and Biomolecular Chemistry, 7, 1753-1760.

[8] Yeo, W.L., Heng, E., Tan, L.L., Lim, Y.W., Lim, Y. H., Hoon, S., Zhao, H., Zhang, M.M., Wong, F.T., 2019. “ Characterization of Cas proteins for CRISPR-Cas editing in stretomycetes.” Biotechnology and Bioengineering, 116.9 2330-2338.

[9] Tong, Y., Charusanti, P., Zhang, L., Weber, T., Lee, S.Y., 2015. "CRISPR-Cas9 based engineering of actinomycetal genomes.” ACS Synthetic Biology, 4.9, 1020-1029.

[10] Zeng, H., Wen, S., Xu, W., He, Z., Zhai, G., Liu, Y., Deng, Z., Sun, Y., 2015. "Highly efficient editing of the actinorhodin polyketide chain length factor gene in Streptomyces coelicolor M145 using CRISPR/Cas9-CodA(sm) combined system.” Applied Microbiology and Biotechnology, $99.24,10575-10585$.

[11] Li, L., Wei, K., Zheng, G., Liu, X., Chen, S., Jiang, W., Lu, Y., 2018. “CRISPR-Cpf1-assisted multiplex genome editing and transcriptional repression in Streptomyces." Applied and Environmental Microbiology, 84.18, e00827-18.

[12] Pausch, P., Al-Shayeb, B., Bisom-Rapp, E., Tsuchida, C.A., Li, Z., Cress, B.F., Knott, G.J., Jacobsen, S.E., Banfield, J.F., Doudna, J.A., 2020. “ CRISPR-Cas $\Phi$ from huge phages is a hypercompact genome editor.” Science, 369.6501, 333-337.

[13] Ng, S. B., Kanagasundaram, Y., Fan, H., Arumugam, P., Eisenhaber, B., \& Eisenhaber, F. (2018). The 160K natural organism library, a unique resource for natural products research. Nature biotechnology, 36(7), 570-573.

[14] Cobb, R.E., Wang, Y., Zhao, H., 2015. "High-efficiency multiplex genome editing of Streptomyces species using an engineered CRISPR/Cas System.” ACS Synthetic Biology, 4.6, 723728. 
[15] Zhang, M.M., Wong, F.T., Wang, Y., Luo, S., Lim, Y.H., Heng, E., Yeo, W.L., Cobb, R.E., Enghiad, B., Ang, E.L., Zhao, H., 2017. "CRISPR-Cas9 strategy for activation of silent Streptomyces biosynthetic gene clusters.” Nature Chemical Biology, 13.6, 607-609.

[16] Heng, E., Tan, L.L., Zhang, M.M., Wong, F.T., 2021. "CRISPR-Cas strategies for natural product discovery and engineering in actinomycetes." Process Biochemistry, 102, 2.

[17] Khomtchouk, B.B., Hennessy, J.R. and Wahlestedt, C., 2017. shinyheatmap: Ultra fast low memory heatmap web interface for big data genomics. PloS one, 12(5), p.e0176334.

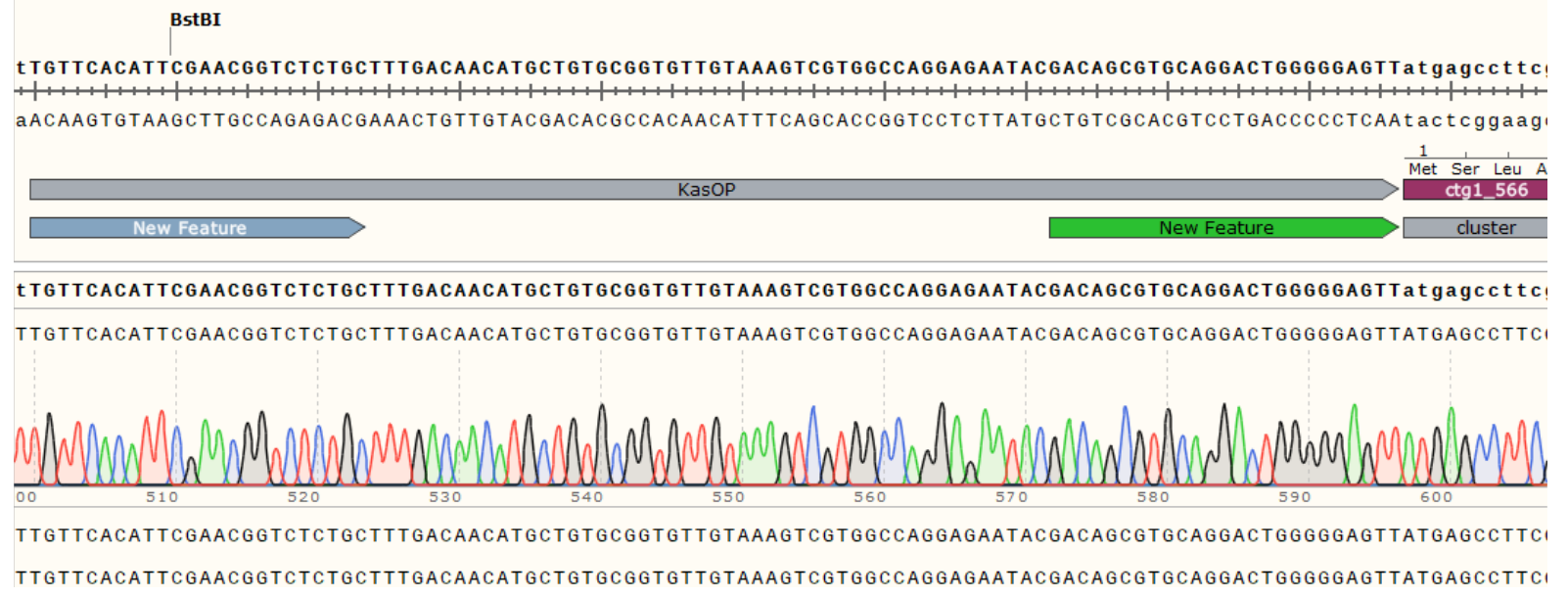

Supplementary Figure 1. Representative trace of edited genome sequence (insertion of $k a s O^{*} \mathrm{p}$ ) for cluster 74 in Streptomyces sp. A34053. 